\title{
Méthode du gradient projeté avec contrôle d'erreur
}

\section{Une méthode simple et efficace en fiabilité des structures}

\author{
Xuan Son Nguyen - Frédéric Duprat - Alain Sellier \\ Gérard Pons
}

Université de Toulouse, Laboratoire Matériaux et Durabilité des Constructions LMDC-UPS/INSA

135 avenue de Rangueil, F-31077 Toulouse cedex

\{frederic.duprat, alain.sellier\}@insa-toulouse.fr

RÉSUMÉ. Le recours aux approches probabilistes est actuellement de plus en plus fréquent dans la conception et l'estimation de la durabilité des structures de génie civil. L'indice de fiabilité de Hasofer-Lind est à ce titre la mesure de fiabilité recommandée par les codes de dimensionnement. Le calcul de cet indice est un problème d'optimisation sous contrainte dont un algorithme itératif simple a été proposé par Rackwitz et Fiessler, basé sur la méthode du gradient projeté. L'inconvénient de cette méthode réside dans ce que l'évaluation du vecteur gradient de la fonction d'état limite est souvent réalisée par différences finies. Si l'incrément choisi pour ce calcul donne une variation de la réponse inférieure à la précision de la fonction d'état limite, l'algorithme peut donner des résultats erronés, voire ne pas converger. Pour pallier cet inconvénient, nous proposons une méthode dite du gradient projeté avec contrôle d'erreur (GRACE). L'algorithme simple proposé ici permet de tenir compte de la précision numérique de la fonction d'état limite par une procédure de choix de l'incrément. Les résultats obtenus montrent la bonne convergence de l'algorithme proposé et son efficacité en termes de nombre d'appels à la fonction de défaillance.

ABSTRACT. Probabilistic approaches are today frequently used in the design of new civil engineering structures and durability analysis of existing constructions. The so-called Hasofer-Lind's reliability index is the most popular reliability measure in design codes. This index can be determined by several minimizations under constraint algorithms, such as Rackwitz-Fiessler's algorithm which is based on the projected gradient method. The drawback of this method lies in the estimation of the gradient vector of the limit state function which is often carried out by finite differences. If the perturbation chosen for this estimation gives a variation of the result lower than the accuracy of the limit state function, the algorithm could give erroneous results, not even to converge. In order to circumvent this drawback, we propose a method called projected gradient method with error control. The principle is to add to Rackwitz-Fiessler's algorithm a procedure for choosing judiciously the perturbation for calculating the gradient vector accounting for the numerical accuracy of the limit state function. The efficiency of the proposed method can be judged from examples taken from the literature.

MOTS-CLÉS : méthodes probabilistes, fiabilité des structure, erreur numérique.

KEYWORDS: probabilistic methods, structural reliability, numerical error.

DOI:10.3166/REMN.17.1039-1056 @ 2008 Lavoisier, Paris

Revue européenne de mécanique numérique. Volume 17 - n 8/2008, pages 1039 à 1056 


\section{Introduction}

Les propriétés mécaniques des matériaux constitutifs des ouvrages de génie civil, et des structures de façon générale, de même que leur géométrie et les actions appliquées présentent des écarts aléatoires par rapport aux valeurs de projet. Dans le cadre de la conception ou de la requalification des structures, la prise en compte de ces incertitudes est une nécessité. Les guides et codes normatifs de dimensionnement réalisent partiellement cette prise en compte, le plus souvent de façon simplifiée. L'approche probabiliste reste cependant l'outil le plus efficace pour une prise en compte rationnelle des aléas dans l'estimation de la sécurité ou de la durabilité des ouvrages. Dans cette approche, un couplage doit être réalisé entre l'outil de modélisation du comportement des ouvrages, le plus performant étant la méthode des éléments finis, et l'algorithme probabiliste proprement dit visant à donner une mesure de la fiabilité structurale. Le couplage consiste d'une part, à probabiliser les paramètres d'entrée du modèle aux éléments finis, et, d'autre part, à intégrer la réponse de ce modèle (déplacements, contraintes, etc.) dans l'expression de la fonction de défaillance traitée par l'algorithme probabiliste. Cependant, les possibilités actuelles de raffinement des modèles mécaniques s'accompagnent d'une inflation des temps de calcul limitant la mise en œuvre pratique de l'approche probabiliste. Le développement d'outils pour l'approche probabiliste permettant de minimiser le temps de calcul en assurant la précision des résultats reste d'actualité.

L'indice de fiabilité d'Hasofer-Lind (Hasofer et Lind, 1974) est fréquemment utilisé comme mesure de la fiabilité structurale. Cet indice est défini dans l'espace des variables aléatoires normales centrées réduites et stochastiquement indépendantes, appelé espace standardisé, comme la distance minimale de l'origine de l'espace gaussien à la surface de défaillance. Un algorithme itératif robuste pour déterminer l'indice d'Hasofer-Lind a été proposé dès 1978 (Rackwitz et Fiessler, 1978). Cet algorithme, ainsi d'ailleurs que les algorithmes qui en ont été dérivés afin de l'améliorer, nécessitent le calcul du vecteur gradient de la fonction de défaillance, composé des dérivées partielles de celle-ci par rapport aux variables standardisées. Lorsque la fonction de défaillance est définie à partir d'un modèle numérique ne fournissant que des approximations de la solution exacte du problème, le calcul du vecteur gradient ne peut à son tour qu'être approché, ce qui peut entraîner des imprécisions, voire même dans certains cas la non convergence de l'algorithme probabiliste. Pour pallier au problème des imprécisions, nous proposons, dans cette étude, une procédure simple et efficace de choix de l'incrément pour évaluer des dérivées partielles de la fonction d'état limite selon la méthode des différences finies. Cette procédure effectuée en tenant compte de la précision numérique du modèle permet d'augmenter la précision des résultats obtenus et de réduire le temps de calcul numérique. 


\section{Détermination de l'indice de fiabilité}

Le calcul de l'indice d'Hasofer-Lind est un problème d'optimisation sous contrainte, consistant à minimiser la norme du vecteur $u$ des variables aléatoires dans l'espace standardisé, sous contrainte de nullité de la fonction de défaillance $G(u)$. Le point solution $P^{*}$ tel que $\beta=\left\|O P^{*}\right\|=\left\|u^{*}\right\|$ est le point de défaillance le plus probable (ou point de conception), soit :

$$
u^{*}=\arg \min (\|u\|)_{G(u)=0}
$$

$G(u)=0$ définit la frontière (surface de défaillance) entre le domaine de performance pour lequel, par convention, $G(u)>0$ et de domaine de défaillance pour lequel $G(u)<0$. Au sens des codes de conception des ouvrages de génie civil, $G(u)$ est une fonction d'état limite, qui est généralement continue dans le domaine de performance, et, au moins à proximité de la surface de défaillance, dans le domaine de défaillance. Cette particularité explique que les algorithmes applicables aux fonctions $G(u)$ différentiables aient été souvent retenus pour la fiabilité mécanique, bien que de nombreux autres algorithmes permettent de résoudre le problème [1] comme l'algorithme du simplexe (Nelder et Mead, 1965), l'algorithme de Polak-He (Polak, 1997) ou plus récemment l'algorithme des faisceaux (Bonnans et al, 1999). L'indice de fiabilité étant une distance euclidienne, il est nécessaire de le déterminer dans un espace probabilisé adimensionnel, où toutes les variables physiques, de lois quelconques éventuellement corrélées, deviennent des variables standardisées (gaussiennes centrées réduites et indépendantes). Le passage d'un espace à l'autre est ici réalisé par les transformations de Nataf (1962).

Rackwitz et Fiessler (1978) ont proposé une méthode itérative, basée sur la méthode du gradient projeté, qui résout le problème [1] en générant une suite de points qui converge vers une solution optimale. Le nouvel itéré $u_{(k+1)}$ à l'itération $(k+1)$ est exprimé par :

$$
u_{(k+1)}=\frac{\nabla G\left(u_{(k)}\right)}{\left\|\nabla G\left(u_{(k)}\right)\right\|^{2}}\left(u_{(k)}^{T} \nabla G\left(u_{(k)}\right)-G\left(u_{(k)}\right)\right)
$$

Dans cette expression, $u_{(k)}^{T} \nabla G\left(u_{(k)}\right)$ est la projection du vecteur $u_{(k)}$ sur la direction du gradient $\nabla G\left(u_{(k)}\right)$, à laquelle on apporte la correction $-G\left(\mathrm{u}_{(k)}\right)$ (figure 1). Le point $P^{*}$ est obtenu lorsque la proximité de deux points consécutifs est satisfaisante, soit pour les coordonnées de l'axe $i$ :

$$
\left|u_{i(k+1)}-u_{i(k)}\right| \leq \varepsilon_{P}
$$


La direction de descente de l'algorithme est définie à chaque itération par :

$$
d_{(k)}=\frac{\nabla G\left(u_{(k)}\right)}{\left\|\nabla G\left(u_{(k)}\right)\right\|^{2}}\left(u_{(k)}^{T} \nabla G\left(u_{(k)}\right)-G\left(u_{(k)}\right)\right)-u_{(k)}
$$

ce qui permet d'écrire le nouvel itéré sous la forme :

$$
u_{(k+1)}=u_{(k)}+\lambda_{(k)} d_{(k)}
$$

où $\lambda_{(k)}$ est le pas de descente, unitaire pour l'algorithme de Rackwitz-Fiessler. Les principales améliorations de la convergence de ce dernier ont porté sur les méthodes permettant d'optimiser le pas de descente $\lambda_{(k)}$. Le pas optimal peut être obtenu en résolvant un nouveau problème d'optimisation à chaque itération, consistant à minimiser l'écart d'une fonction de mérite entre deux itérés successifs, comme l'ont proposé Zhang et Der Kiureghian (1995).

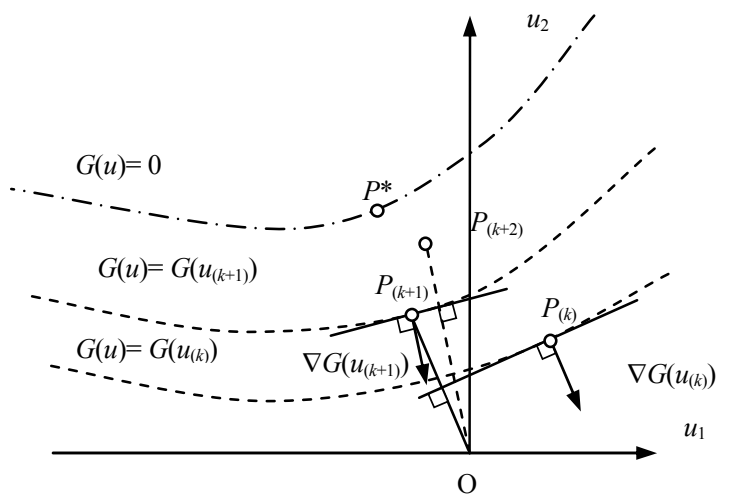

Figure 1. Progression de l'algorithme de Rackwitz-Fiessler

En alternative aux algorithmes dérivés de la méthode du gradient projeté, des algorithmes ont été développés, basés sur l'expression du lagrangien associé au problème [1], soit

$$
L(u, \gamma)=f(u)+\gamma G(u)
$$

où $f(u)$ est la fonction objectif. La recherche de la solution annulant le gradient de $L(u, \gamma)$ sous la condition $G(u)=0$ peut être obtenue de façon séquentielle, en linéarisant ce dernier au second ordre par une série de Taylor. C'est le principe des méthodes dites Sequential Quadratic Programming (SQP). Le calcul de la matrice 
hessienne $\nabla^{2} G(u)$ est nécessaire dans ce schéma quasi-Newton. Liu et Der Kiureghian (1991) ont proposé un algorithme dans lequel cette matrice est affinée au fur et à mesure des itérations, avec une fonction objectif $f(u)=\|u\|^{2} / 2$.

Abdo et Rackwitz (1991) ont utilisé le lagrangien augmenté

$$
L(u, \gamma)=f(u)+\gamma G(u)+c G(u)^{2} / 2
$$

d'une fonction objectif de la forme $f(u)=\|u\|^{2}$, comme fonction de mérite pour déterminer le pas optimal de descente dans l'algorithme de Rackwitz-Fiessler. Ce choix simplifie le schéma SQP par réduction de la matrice hessienne à la matrice diagonale unité.

Les algorithmes brièvement décrits ici sont globalement convergents, et en général plus robustes que l'algorithme de Rackwitz-Fiessler. Cependant ils ont recours, comme ce dernier, au gradient $\nabla G(u)$ de la fonction de défaillance et leur efficacité dépend donc de la qualité de son estimation. La fonction de défaillance étant définie par rapport aux variables physiques, il est nécessaire de calculer les dérivées partielles dans l'espace standardisé à partir des dérivées partielles dans l'espace physique, soit :

$$
\frac{\partial G(u)}{\partial u_{i}}=\nabla G(u)_{i}=\nabla G(x)^{T} \frac{\partial x}{\partial u_{i}}=\nabla G(x)^{T} \frac{\partial T^{-1}(u)}{\partial u_{i}}
$$

où $\frac{\partial G(u)}{\partial u_{i}}$ est la dérivée partielle de la fonction d'état limite par rapport à la variable normée $u_{\mathrm{i}}, u=T(x)$ permet le passage de l'espace physique vers l'espace standardisé, $\nabla G(x)$ est le gradient de la fonction d'état limite par rapport aux variables physiques $x$. Dans de nombreux problèmes pratiques, une formulation explicite du gradient de la fonction d'état limite par rapport aux variables physiques n'est pas disponible. Il est donc nécessaire de recourir aux calculs par différence finie de la dérivée partielle :

$$
\frac{\partial G(x)}{\partial x_{i}}=\nabla G(x)_{i} \approx \frac{G\left(x+\Delta x_{i} e_{i}\right)-G(x)}{\Delta x_{i}}
$$

où $\Delta x_{i}$ est un incrément selon l'axe $x_{i}$ et $e_{i}$ est le vecteur unitaire de la base physique pour la variable $x_{i}$. Pour limiter l'erreur de troncature liée à l'approximation [9], la tentation est grande de choisir un incrément $\Delta x_{i}$ faible. Cependant, ce choix peut conduire à la non-convergence des algorithmes, du fait de l'incompatibilité entre la taille de l'incrément et la précision du modèle. En effet la réponse des modèles numériques non linéaires présente toujours une partie déterministe et une partie 
aléatoire proportionnelle aux imprécisions numériques admises dans les algorithmes de résolution des procédures non linéaires, notée $\Delta G(x)$ sur la figure 2.

Ainsi, si l'incrément $\Delta x_{i}$ est trop faible $\left(\Delta x_{i}<\Delta x_{\min }\right)$, la différence des réponses du modèle entre les deux bornes de l'incrément est aléatoire. Pour estimer correctement une différence finie il faut donc imposer à l'incrément une valeur suffisamment grande pour que la composante aléatoire de la différence des réponses devienne négligeable. De plus, il est illusoire de réestimer une différence finie dans une direction de l'espace tant que le point courant de la procédure itérative ne s'est pas suffisamment éloigné de l'endroit où l'on avait estimé la précédente différence finie.

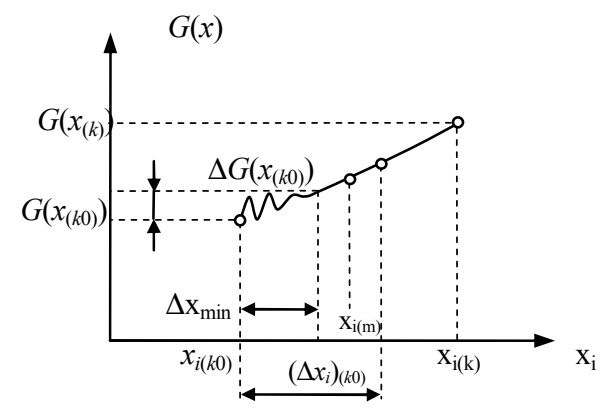

Figure 2. Problème posé par la valeur de l'incrément $\Delta x_{i}$

Pour éviter l'écueil de l'estimation du gradient, l'emploi d'une surface de réponse explicite et deux fois différentiable, approchant la réponse de la fonction de défaillance implicite, est également possible. La construction itérative de la surface de réponse, au fur et à mesure de la progression vers le point $P^{*}$, assure généralement un comportement satisfaisant de celle-ci au voisinage de ce point, mais cette construction reste d'un coût numérique élevé en grande dimension, avec par exemple plus de 20 variables (Duprat et Sellier, 2006 ; Nguyen et al., 2007).

\section{Procédure pour choisir l'incrément lors de l'estimation du gradient}

Pour prendre en compte de façon «automatique» les considérations mentionnées ci-avant, nous proposons d'intégrer dans l'algorithme de RackwitzFiessler une procédure combinant le gradient de l'itération précédente et la précision numérique du modèle de défaillance pour calculer la taille minimale des incréments à utiliser dans l'estimation du nouveau gradient. Le choix de l'incrément est effectué en espace physique.

Supposons que la fonction d'état limite soit définie comme la différence entre la résistance $R(x)$ de la structure et la sollicitation appliquée $S(x)$, soit : 


$$
G(x)=R(x)-S(x)
$$

La part aléatoire des réponses, ou imprécision numérique, est proportionnelle aux réponses des modèles, avec des coefficients respectifs $\varepsilon_{R}$ et $\varepsilon_{S}$. Pour la première itération, la sensibilité du modèle aux différentes variables n'étant pas connue, l'incrément est choisi arbitrairement très grand :

$$
\Delta x_{i}=c_{1}\left(\varepsilon_{R}+\varepsilon_{S}\right) x_{i}
$$

où $c_{1}$ est un coefficient d'amplification $\left(c_{1}>1\right.$, on propose $\left.c_{1}=10\right)$. Pour l'itération courante $(k), \Delta x_{i}$ est calculé par :

$$
\left(\Delta x_{i}\right)_{(k)}=c_{2}\left|\frac{\Delta G\left(x_{(k-1)}\right)}{\nabla G\left(x_{(k-1)}\right)_{i}}\right|=c_{2}\left|\frac{\varepsilon_{R} R\left(x_{(k-1)}\right)+\varepsilon_{S} S\left(x_{(k-1)}\right)}{\nabla G\left(x_{(k-1)}\right)_{i}}\right|
$$

où $c_{2}$ est un coefficient d'amplification $\left(c_{2}>1\right.$, on propose $\left.c_{2}=10\right)$, et $\Delta G\left(x_{(k-1)}\right)=\varepsilon_{R} R\left(x_{(k-1)}\right)+\varepsilon_{S} S\left(x_{(k-1)}\right) \quad$ est l'imprécision numérique maximale possible sur la fonction de défaillance.

Comme remarqué précédemment, il peut arriver que deux points consécutifs de la procédure itérative de Rackwitz-Fiessler se trouvent, pour un axe particulier, à une distance inférieure à celle requise pour évaluer correctement la dérivée partielle. Dans ce cas, nous préconisons de ne pas réactualiser la dérivée partielle tant que la distance parcourue sur l'axe n'est pas supérieure à celle requise pour évaluer la dérivée partielle. La condition pour actualiser une composante $\nabla G\left(x_{(k)}\right)_{i}$ du gradient est donc (figure 2) :

$$
\left|\sum_{m=k_{0}+1}^{k}\left(\left(x_{i}\right)_{(m)}-\left(x_{i}\right)_{(m-1)}\right)\right| \geq\left(\Delta x_{i}\right)_{\left(k_{0}\right)}
$$

où $\left(\left(x_{i}\right)_{(m)}-\left(x_{i}\right)_{(m-1)}\right)$ est la distance algébrique mesurée sur l'axe $x_{i}$ entre deux dernières itérations de l'algorithme de Rackwitz-Fiessler, $\left(k_{0}\right)$ est le numéro d'itération lors du dernier calcul de la dérivée partielle pour l'axe $x_{i}$ et $(k)$ le numéro de l'itération actuelle. La condition [13] permet d'économiser les évaluations de la fonction d'état limite selon les axes pour lesquels l'optimum est atteint. Au final, le choix des incréments en fonction de l'imprécision numérique possible sur le modèle, combiné à cette technique limitant le nombre de réévaluations des dérivées partielles, conduit à favoriser et à accélérer la convergence de l'algorithme. 


\section{Exemples}

Nous donnons ci-après quatre exemples pour illustrer l'efficacité de la méthode proposée, et le gain en coût numérique apporté par le fait de ne pas réévaluer systématiquement les dérivées partielles.

Les deux premiers exemples, portant sur des fonctions de défaillance explicites, offrent une comparaison précise avec d'autres algorithmes. Les résultats présentés sont la valeur de l'indice de fiabilité, les coordonnées du point de conception $P^{*}$, la valeur de la fonction d'état limite en ce point relative à la valeur au point médian $G\left(u^{*}\right) / G\left(u_{0}\right)$ qui vaut zéro si $P^{*}$ se trouve exactement sur la surface de défaillance, et le nombre de calculs de la fonction d'état limite $\mathrm{N}_{\mathrm{c}}$ (indicateur du temps de calcul associé à la méthode). Dans les deux derniers exemples, portant sur des fonctions d'état limite implicite, définies à l'aide de modèles aux éléments finis, les résultats obtenus par GRACE sont comparés aux résultats, parfois incomplets, issus de la littérature.

\subsection{Exemple 1}

Les algorithmes suivants ont été utilisés : algorithmes de Liu-Der Kiureghian (SQP), de Zhang-Der Kiureghian (iHLRF), implantés dans le logiciel libre OpenSees (OPenSees, 2008), (Haukaas et Der Kiureghian, 2006), algorithmes d'Abdo-Rackwitz (RFLS), de Rackwitz-Fiessler (HLRF) et de Nelder-Mead (SPLX), implantés dans le logiciel Comrel en version libre (Comrel, 2008). La fonction d'état limite est une surface de réponse polynômiale utilisée dans l'analyse de fiabilité d'un oléoduc (Liu et Der Kiureghian, 1991) :

$$
\begin{aligned}
& G(x)=1,1-0,00534 x_{1}-0,0705 x_{2}-0,226 x_{3}+0,998 x_{4} \\
& -0,00115 x_{1} x_{2}-0,0149 x_{1} x_{3}+0,0717 x_{1} x_{4}+0,0135 x_{2} x_{3} \\
& -0,0611 x_{2} x_{4}-0,558 x_{3} x_{4}+0,00117 x_{1}^{2}+0,00157 x_{2}^{2} \\
& +0,0333 x_{3}^{2}-1,339 x_{4}^{2}
\end{aligned}
$$

Les variables aléatoires non corrélées sont définies dans le tableau 1. Le critère de convergence et les précisions numériques sont fixés dans GRACE à $\varepsilon_{\mathrm{p}}=510^{-3}$, $\varepsilon_{\mathrm{R}}=\varepsilon_{\mathrm{S}}=10^{-7}$. Le critère de convergence est identique dans Comrel. Dans OpenSees, les critères de convergence sont

$$
\left|G\left(u^{*}\right) / G\left(u_{0}\right)\right| \leq \varepsilon_{1}
$$

vérifiant la proximité à la surface de défaillance de $P^{*}$, et

$$
\left\|u^{*}-\alpha^{* T} u^{*} \alpha^{*}\right\| \leq \varepsilon_{2}
$$

vérifiant que $u^{*}$ est porté par la direction du gradient. Les valeurs par défaut des critères sont $\varepsilon_{1}=\varepsilon_{2}=10^{-3}$. Les résultats sont portés dans les tableaux 2 et 3 . 


\begin{tabular}{|c|c|c|c|}
\hline Variable & Distribution & Moyenne & Ecart type \\
\hline$x_{1}$ & Lognormale & 10 & 5 \\
$x_{2}$ & Normale & 25 & 0,8 \\
$x_{3}$ & Normale & 5 & 0,2 \\
$x_{4}$ & Lognormale & 0,0625 & 0,0625 \\
\hline
\end{tabular}

Tableau 1. Caractéristiques des variables aléatoires - Exemple 1

\begin{tabular}{|c|c|c|c|c|c|}
\hline Technique & $\beta$ & $\mathrm{N}_{\mathrm{c}}$ & $\left|G\left(u^{*}\right) / G\left(u_{0}\right)\right|$ & $\left\|u^{*}-\alpha^{* T} u^{*} \alpha^{*}\right\|$ & $\max \left|u_{i}^{*}-u_{i\left(k^{*}-1\right)}\right|$ \\
\hline SQP & 1,5296 & 139 & $2,7510^{-6}$ & $7,7710^{-4}$ & - \\
iHLRF & 1,5306 & 45 & $1,2410^{-4}$ & $7,0610^{-4}$ & - \\
HLRF & 1,5296 & 25 & $2,1010^{-7}$ & - & $1,5810^{-3}$ \\
RFLS & 1,5296 & 25 & $2,1010^{-7}$ & - & $1,5810^{-3}$ \\
SPLX & 1,5294 & 49 & $1,1410^{-6}$ & - & $2,1110^{-3}$ \\
GRACE & 1,5296 & 23 & $1,8410^{-7}$ & $6,5110^{-7}$ & $1,7010^{-3}$ \\
\hline
\end{tabular}

Tableau 2. Indice de fiabilité et convergence - Exemple 1

\begin{tabular}{|c|c|c|c|c|}
\hline Technique & $u^{*}{ }_{1}$ & $u^{*}{ }_{2}$ & $u^{*}{ }_{3}$ & $u^{*}{ }_{4}$ \\
\hline SQP & 1,499 & $-0,115$ & $-0,080$ & 0,267 \\
iHLRF & 1,509 & $-0,152$ & $-0,079$ & 0,214 \\
HLRF & 1,496 & $-0,114$ & $-0,079$ & 0,284 \\
RFLS & 1,496 & $-0,114$ & $-0,079$ & 0,284 \\
SPLX & 1,495 & $-0,118$ & $-0,076$ & 0,284 \\
GRACE & 1,496 & $-0,114$ & $-0,079$ & 0,284 \\
\hline
\end{tabular}

Tableau 3. Coordonnées de $P^{*}$ - Exemple 1

Les résultats sont très proches les uns des autres, en ce qui concerne la valeur de l'indice de fiabilité et le point de conception $P^{*}$. En ce qui concerne le coût numérique, les algorithmes les plus séduisants sont ceux basés sur la méthode du gradient projeté avec optimisation a minima du pas de descente (RFLS, HLRF, GRACE). Ces trois algorithmes convergent en 5 itérations vers un point très proche de la surface de défaillance. Sur cet exemple RFLS et HLRF donnent rigoureusement les mêmes résultats. Par économie de deux calculs de dérivées partielles, GRACE est l'algorithme le moins couteux. On note que le critère de convergence [3], retenu dans GRACE, est plus sévère que les critères [15] et [16] 
des algorithmes iHLRF et SQP. Il convient également de remarquer pour ces deux derniers, qui ont recours à de nombreux calculs complémentaires du gradient pour l'optimisation du pas de descente, que la convergence est très sensible à la finesse de l'incrément choisi pour estimer le gradient par différences finies. Dans OpenSees, la perturbation est imposée en fraction d'écart type : les résultats correspondent ici à une fraction de 1/1000 pour SQP et 1/5000 pour iHLRF. Si une fraction de 1/1000 (valeur par défaut) est choisie pour iHLRF, le nombre de calculs de la fonction d'état limite devient $\mathrm{N}_{\mathrm{c}}=244$ pour des résultats identiques. Enfin, l'algorithme du simplexe SPLX ne présente pas une convergence très efficace : de façon générale, le nombre d'appels à la fonction de défaillance est 2 à 3 fois plus important que pour les algorithmes RFLS ou HLRF (Comrel, 2008).

\subsection{Exemple 2}

Les algorithmes RFLS et HLRF sont retenus pour la comparaison dans cet exemple. La fonction d'état limite est relative à la résistance en flexion d'une section de poutre en béton armé (Madsen et al., 1986) :

$$
G(x)=x_{2} x_{3} x_{4}-\frac{x_{5} x_{3}^{2} x_{4}^{2}}{x_{6} x_{7}}-x_{1}
$$

Les variables aléatoires non corrélées sont définies dans le tableau 4. Les résultats sont portés dans les tableaux 5 et 6 .

\begin{tabular}{|c|c|c|c|c|}
\hline Variable & Désignation & Distribution & Moyenne & Ecart type \\
\hline$x_{1}$ & Moment appliqué (MN.m) & Normale & 0,01 & 0,003 \\
$x_{2}$ & Hauteur utile (m) & Normale & 0,30 & 0,015 \\
$x_{3}$ & Limite élastique acier (MPa) & Normale & 360 & 36 \\
$x_{4}$ & Section d'armatures (m $\left.{ }^{2}\right)$ & Normale & $22610^{-6}$ & $11,310^{-6}$ \\
$x_{5}$ & Coefficient de répartition & Normale & 0,5 & 0,05 \\
$x_{6}$ & Largeur de section (m) & Normale & 0,12 & 0.006 \\
$x_{7}$ & Résistance béton (MPa) & Normale & 40 & 6 \\
\hline
\end{tabular}

Tableau 4. Caractéristiques des variables aléatoires - Exemple 2

\begin{tabular}{|c|c|c|c|c|c|}
\hline Technique & $\beta$ & $\mathrm{N}_{\mathrm{c}}$ & $\left|G\left(u^{*}\right) / G\left(u_{0}\right)\right|$ & $\mid u^{*}-\alpha^{* T} u^{*} \alpha^{*} \|$ & $\max \left|u_{i}^{*}-u_{i\left(k^{*}-1\right)}\right|$ \\
\hline HLRF & 3,4117 & 40 & $6,9010^{-6}$ & - & - \\
RFLS & 3,4132 & 32 & $2,3710^{-7}$ & - & $3,5210^{-3}$ \\
GRACE & 3,4134 & 26 & $6,1310^{-8}$ & $2,8710^{-7}$ & $3,8710^{-3}$ \\
\hline
\end{tabular}

Tableau 5. Indice de fiabilité et convergence - Exemple 2 


\begin{tabular}{|c|c|c|c|c|c|c|c|}
\hline Technique & $u^{*}{ }_{1}$ & $u^{*}{ }_{2}$ & $u^{*}{ }_{3}$ & $u^{*}{ }_{4}$ & $u^{*}{ }_{5}$ & $u_{6}{ }_{6}$ & $u^{*_{7}}$ \\
\hline HLRF & 2,615 & $-0,831$ & $-1,866$ & $-0,790$ & 0,037 & $-0,019$ & $-0,056$ \\
RFLS & 2,606 & $-0,851$ & $-1,864$ & $-0,808$ & 0,039 & $-0,019$ & $-0,059$ \\
GRACE & 2,616 & $-0,828$ & $-1,868$ & $-0,787$ & 0,060 & $-0,030$ & $-0,090$ \\
\hline
\end{tabular}

Tableau 6. Coordonnées de $P^{*}$ - Exemple 2

Les résultats indiqués pour HLRF sont donnés dans (Madsen et al., 1986). Les coordonnées du point $P^{*}$ et la valeur de $\beta$ obtenues sont très proches. GRACE converge de façon satisfaisante, et présente une meilleure efficacité en termes de coût numérique que RFLS : 14 appels à la fonction d'état limite sont en effet économisés par non-réévaluation des dérivées partielles pour les variables peu influentes. Dans le tableau 7 sont indiquées les évaluations des dérivées partielles de la fonction d'état limite par rapport aux variables aléatoires.

\begin{tabular}{|c|c|c|c|c|c|c|}
\hline \multicolumn{2}{|c|}{ Itération } & 1 & 2 & 3 & 4 & 5 \\
\hline \multirow{6}{*}{ Variable } & $u_{1}$ & oui & oui & oui & oui & oui \\
& $u_{2}$ & oui & oui & oui & non & non \\
& $u_{3}$ & oui & oui & oui & oui & non \\
& $u_{4}$ & oui & oui & oui & non & non \\
& $u_{5}$ & oui & oui & non & non & non \\
& $u_{6}$ & oui & oui & non & non & non \\
& $u_{7}$ & oui & oui & non & non & non \\
\hline
\end{tabular}

Tableau 7. Evaluation des dérivées partielles - Exemple 2

Dès la $3^{\mathrm{e}}$ itération, GRACE ne réévalue pas les dérivées partielles de la fonction d'état limite par rapport aux variables $u_{5}, u_{6}, u_{7}$ et prend leurs valeurs à l'itération 2 . La procédure associant un choix rationnel de l'incrément pris sur les axes pour l'évaluation des dérivées partielles à un critère de décision pour le calcul du gradient, confère à l'algorithme la possibilité « d'oublier » les variables peu influentes, de façon similaire à l'emploi des facteurs d'omission (Madsen, 1988). Le facteur d'omission est défini pour une variable aléatoire comme le rapport de l'indice calculé en fixant la variable à sa valeur médiane, à l'indice de fiabilité. Lorsque ce rapport est proche de l'unité, la variable est significativement non influente. Le tableau 8 présente les valeurs du facteur d'omission $\gamma_{i}\left(\mu_{i}\right)$ associé à $x_{i}$. Les résultats montrent que l'influence des variables $x_{5}, x_{6}$ et $x_{7}$ est très faible. L'intégration directe des facteurs d'omission dans l'algorithme de recherche de l'indice de fiabilité a été proposée sur la base d'une estimation approchée de ceux-ci (Madsen, 1988). Cependant, tandis que cette dernière nécessite un complément algorithmique et présente un caractère définitif 
dans l'algorithme, GRACE intègre de façon indirecte les facteurs d'omission, sans complément algorithmique particulier et sans que cela soit irrévocable : c'est là un intérêt supplémentaire à la méthode.

\begin{tabular}{|c|c|c|c|c|c|c|c|}
\hline Variable & $x_{1}$ & $x_{2}$ & $x_{3}$ & $x_{4}$ & $x_{5}$ & $x_{6}$ & $x_{7}$ \\
\hline$\beta$ & 5,5614 & 3,5151 & 4,0203 & 3,5052 & 3,4133 & 3,4132 & 3,4134 \\
\hline$\gamma_{i}\left(\mu_{i}\right)$ & 1,629 & 1,029 & 1,778 & 1,026 & 0,999 & 0,999 & 1,000 \\
\hline
\end{tabular}

Tableau 8. Facteurs d'omission - Exemple 2

\subsection{Exemple 3}

Afin de montrer l'efficacité de GRACE pour des problèmes à grand nombre de variables aléatoires, l'exemple suivant est proposé. Il s'agit d'un portique à 3 travées et 5 étages (Liu et Der Kiureghian, 1986). La structure est schématisée sur la figure 3. Elle est caractérisée par 21 variables de base. Les données structurales et leurs propriétés statistiques sont présentées dans le tableau 9 et le tableau 10. Des coefficients de corrélation sont considérés, entre les charges $\left(\rho_{\mathrm{FiFj}}=0,95\right)$, entre les propriétés géométriques $\left(\rho_{\mathrm{AiAj}}=\rho_{\mathrm{Iijj}} \rho_{\mathrm{Ailj}}=0,13\right)$ ainsi qu'entre les modules d'élasticité $\left(\rho_{\mathrm{E} 1 \mathrm{E} 2}=0,9\right)$. Les autres variables sont supposées être non corrélées.

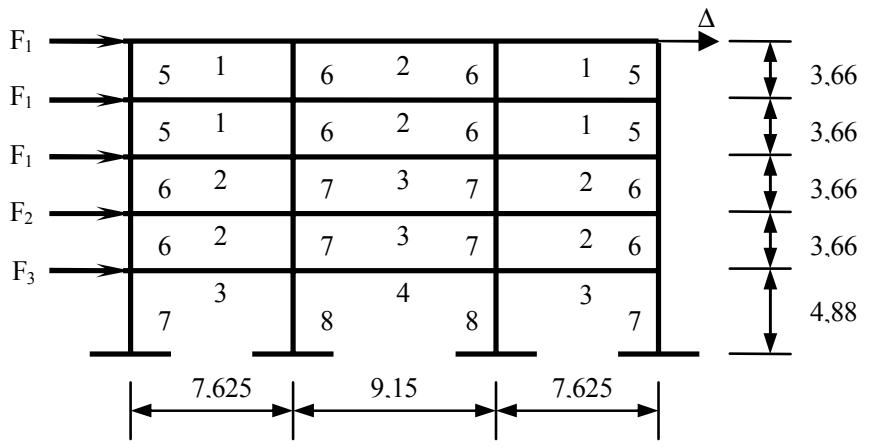

Figure 3. Portique de l'exemple 3 (unités : $m$ )

Le critère de défaillance concerne l'état limite de service en déplacement $\mathrm{du}$ portique : il s'agit du dépassement du déplacement horizontal au sommet du portique $\Delta$ par rapport à un seuil de $0,061 \mathrm{~m}$. La fonction d'état limite est alors implicitement définie par :

$$
G\left(x_{1}, \ldots, x_{21}\right)=0,061-\Delta\left(x_{1}, \ldots, x_{21}\right)
$$




\begin{tabular}{|c|c|c|c|}
\hline Elément & Module d'élasticité & Moment d'inertie & Section \\
\hline 1 & $\mathrm{E}_{1}$ & $\mathrm{I}_{5}$ & $\mathrm{~A}_{5}$ \\
2 & $\mathrm{E}_{1}$ & $\mathrm{I}_{6}$ & $\mathrm{~A}_{6}$ \\
3 & $\mathrm{E}_{1}$ & $\mathrm{I}_{7}$ & $\mathrm{~A}_{7}$ \\
4 & $\mathrm{E}_{1}$ & $\mathrm{I}_{8}$ & $\mathrm{~A}_{8}$ \\
5 & $\mathrm{E}_{2}$ & $\mathrm{I}_{1}$ & $\mathrm{~A}_{1}$ \\
6 & $\mathrm{E}_{2}$ & $\mathrm{I}_{2}$ & $\mathrm{~A}_{2}$ \\
7 & $\mathrm{E}_{2}$ & $\mathrm{I}_{3}$ & $\mathrm{~A}_{3}$ \\
8 & $\mathrm{E}_{2}$ & $\mathrm{I}_{4}$ & $\mathrm{~A}_{4}$ \\
\hline
\end{tabular}

Tableau 9. Propriété des éléments du portique - Exemple 3

\begin{tabular}{|c|c|c|c|}
\hline Variable & Distribution & Valeur moyenne & Ecart type \\
\hline $\mathrm{F}_{1}(\mathrm{kN})$ & Gumbel max & 133,454 & 40,04 \\
$\mathrm{~F}_{2}(\mathrm{kN})$ & Gumbel max & 88,97 & 35,59 \\
$\mathrm{~F}_{3}(\mathrm{kN})$ & Gumbel max & 71,175 & 28,47 \\
$\mathrm{E}_{1}\left(\mathrm{kN} / \mathrm{m}^{2}\right)$ & Normale & $2,173752 \times 10^{7}$ & $1,9152 \times 10^{6}$ \\
$\mathrm{E}_{2}\left(\mathrm{kN} / \mathrm{m}^{2}\right)$ & Normale & $2,379636 \times 10^{7}$ & $1,9152 \times 10^{6}$ \\
$\mathrm{I}_{1}\left(\mathrm{~m}^{4}\right)$ & Normale & $0,813443 \times 10^{-2}$ & $1,08344 \times 10^{-3}$ \\
$\mathrm{I}_{2}\left(\mathrm{~m}^{4}\right)$ & Normale & $1,150936 \times 10^{-2}$ & $1,298048 \times 10^{-3}$ \\
$\mathrm{I}_{3}\left(\mathrm{~m}^{4}\right)$ & Normale & $2,137452 \times 10^{-2}$ & $2,59609 \times 10^{-3}$ \\
$\mathrm{I}_{4}\left(\mathrm{~m}^{4}\right)$ & Normale & $2,596095 \times 10^{-2}$ & $3,028778 \times 10^{-3}$ \\
$\mathrm{I}_{5}\left(\mathrm{~m}^{4}\right)$ & Normale & $1,081076 \times 10^{-2}$ & $2,596095 \times 10^{-3}$ \\
$\mathrm{I}_{6}\left(\mathrm{~m}^{4}\right)$ & Normale & $1,410545 \times 10^{-2}$ & $3,46146 \times 10^{-3}$ \\
$\mathrm{I}_{7}\left(\mathrm{~m}^{4}\right)$ & Normale & $2,327853 \times 10^{-2}$ & $5,624873 \times 10^{-3}$ \\
$\mathrm{I}_{8}\left(\mathrm{~m}^{4}\right)$ & Normale & $2,596095 \times 10^{-2}$ & $6,490238 \times 10^{-3}$ \\
$\mathrm{~A}_{1}\left(\mathrm{~m}^{2}\right)$ & Normale & 0,312564 & 0,055815 \\
$\mathrm{~A}_{2}\left(\mathrm{~m}^{2}\right)$ & Normale & 0,3721 & 0,07442 \\
$\mathrm{~A}_{3}\left(\mathrm{~m}^{2}\right)$ & Normale & 0,50606 & 0,093025 \\
$\mathrm{~A}_{4}\left(\mathrm{~m}^{2}\right)$ & Normale & 0,55815 & 0,11163 \\
$\mathrm{~A}_{5}\left(\mathrm{~m}^{2}\right)$ & Normale & 0,253028 & 0,093025 \\
$\mathrm{~A}_{6}\left(\mathrm{~m}^{2}\right)$ & Normale & 0,29116825 & 0,1023275 \\
$\mathrm{~A}_{7}\left(\mathrm{~m}^{2}\right)$ & Normale & 0,37303 & 0,1209325 \\
$\mathrm{~A}_{8}\left(\mathrm{~m}^{2}\right)$ & Normale & 0,4186 & 0,195375 \\
\hline
\end{tabular}

Tableau 10. Caractéristiques des variables aléatoires - Exemple 3

Le critère de convergence et les précisions numériques sont fixés dans GRACE à $\varepsilon_{\mathrm{p}}=510^{-2}, \varepsilon_{\mathrm{R}}=10^{-7}$ et $\varepsilon_{\mathrm{S}}=10^{-5}$. La valeur de $\varepsilon_{\mathrm{S}}$ est celle utilisée dans le logiciel libre Castem (2005) pour le calcul du déplacement $\Delta$. Les techniques iHLRF, SQP et HLRF ont été utilisées dans la référence (Liu et Der Kiureghian, 1991), dans laquelle seul le nombre $\mathrm{N}_{\mathrm{c}}$ est indiqué, valant respectivement 198, 160 et 264 pour 
chacune de ces techniques. Bucher et Bourgund (1990) ont eu recours à une méthode de surface de réponse adaptative pour laquelle l'indice de fiabilité, estimé $\operatorname{par} \beta=\Phi^{-1}\left(P_{f}\right)$ où $P_{f}$ est la probabilité de défaillance et $\Phi$ la fonction de répartition de la loi normale centrée réduite, vaut 3,290 , avec $\mathrm{N}_{\mathrm{c}}=87$ (requis pour la construction de la surface de réponse). Les résultats obtenus par GRACE sont présentés dans le tableau 11.

\begin{tabular}{|c|c|c|c|c|c|}
\hline Technique & $\beta$ & $\mathrm{N}_{\mathrm{c}}$ & $\left|G\left(u^{*}\right) / G\left(u_{0}\right)\right|$ & $\left\|u^{*}-\alpha^{* T} u^{*} \alpha^{*}\right\|$ & $\max \left|u_{i}^{*}-u_{i\left(k^{*}-1\right)}\right|$ \\
\hline GRACE & 3,211 & 86 & $1,4010^{-5}$ & $9,9310^{-6}$ & $3,3710^{-2}$ \\
\hline
\end{tabular}

Tableau 11. Indice de fiabilité et convergence - Exemple 3

\begin{tabular}{|c|c|c|c|c|c|c|}
\hline \multicolumn{2}{|c|}{ Itération } & 1 & 2 & 3 & 4 & 5 \\
\hline \multirow{6}{*}{} & $u_{1}$ & oui & oui & oui & oui & oui \\
& $u_{2}$ & oui & oui & oui & oui & oui \\
& $u_{3}$ & oui & oui & oui & oui & oui \\
& $u_{4}$ & oui & oui & oui & oui & oui \\
& $u_{5}$ & oui & oui & oui & oui & oui \\
& $u_{6}$ & oui & oui & oui & oui & non \\
& $u_{7}$ & oui & oui & oui & oui & oui \\
& $u_{8}$ & oui & oui & oui & oui & oui \\
& $u_{9}$ & oui & oui & oui & oui & oui \\
& $u_{10}$ & oui & oui & oui & oui & oui \\
& $u_{11}$ & oui & oui & non & oui & oui \\
& $u_{12}$ & oui & oui & oui & oui & oui \\
& $u_{13}$ & oui & oui & oui & oui & oui \\
& $u_{14}$ & oui & oui & non & non & non \\
& $u_{15}$ & oui & oui & non & non & non \\
& $u_{16}$ & oui & oui & oui & oui & non \\
& $u_{17}$ & oui & oui & non & non & non \\
& $u_{18}$ & oui & oui & non & non & non \\
& $u_{19}$ & oui & oui & non & non & non \\
& $u_{20}$ & oui & oui & non & non & non \\
& $u_{21}$ & oui & oui & non & non & non \\
\hline
\end{tabular}

Tableau 12. Evaluation des dérivées partielles - Exemple 3

En termes de convergence, ces résultats sont satisfaisants : la proximité de $P^{*}$ à la surface de défaillance est acceptable de même que la direction du gradient. En ce qui concerne l'efficacité, on constate que GRACE requiert moins d'appels à la fonction de défaillance que les autres techniques, ce qui lui confère un coût numérique moindre. L'économie de 24 appels réalisée par non-réévaluation des dérivées partielles est portée dans le tableau 12. On constate qu'il est possible que la procédure de calcul des incréments «oublie» une variable lors d'une itération, pour la considérer aux 
itérations suivantes, lorsque le critère [13] est à nouveau vérifié. C'est le cas par exemple de la variable $u_{11}$. Cet exemple montre que la technique GRACE reste précise pour un problème à grand nombre de variables corrélées, et permet de réduire le coût numérique par non-réévaluation adaptative des dérivées partielles.

\subsection{Exemple 4}

Dans cet exemple, nous considérons une poutre en béton armé exposée à la carbonatation atmosphérique, précédemment analysée dans (Duprat et Sellier, 2006). La fonction d'état limite correspond à l'atteinte des armatures par le front de carbonatation, permettant une initiation de la corrosion des armatures :

$$
G\left(x_{1}, \ldots, x_{5}\right)=x_{1}-d_{c}\left(x_{2}, \ldots, x_{5}\right)
$$

où $d_{c}\left(x_{2}, . ., x_{5}\right)$ est la profondeur carbonatée calculée par un modèle aux éléments finis 2D. Ce dernier résout l'équation de diffusion du dioxyde de carbone, établie à température et humidité relative supposées constantes, et dans laquelle existe une forte non-linéarité entre la pression de dioxyde de carbone et le terme de puits (la précipitation de la calcite). Cette non-linéarité est ainsi reportée dans la réponse de la fonction de défaillance. Les caractéristiques des variables aléatoires sont présentées dans le tableau 13. Le critère de convergence et les précisions numériques sont fixés dans GRACE à $\varepsilon_{\mathrm{p}}=510^{-2}, \varepsilon_{\mathrm{R}}=10^{-7}$ et $\varepsilon_{\mathrm{S}}=10^{-5}$.

\begin{tabular}{|c|c|c|c|c|}
\hline Variable & Désignation & Distribution & Moyenne & Ecart type \\
\hline$x_{1}$ & Enrobage $(\mathrm{cm})$ & Lognormale & 2 & 0,4 \\
$x_{2}$ & Diffusivité du CO $\left(\mathrm{m}^{2} / \mathrm{s}\right)$ & Lognormale & $10^{-8}$ & $0,810^{-8}$ \\
$x_{2}$ & Tortuosité & Uniforme & 0,5 & 0,23 \\
$x_{4}$ & Résistance du béton $(\mathrm{MPa})$ & Lognormale & 35 & 5 \\
$x_{5}$ & Charge appliquée $\left(\mathrm{kN} / \mathrm{m}^{2}\right)$ & Gumbel Max & 1,04 & 0,4 \\
\hline
\end{tabular}

Tableau 13. Caractéristiques des variables aléatoires - Exemple 4

Le Tableau 14 et le Tableau 15 montrent les résultats obtenus pour la carbonatation de la poutre à une échéance de 5 ans. L'indice de fiabilité $\beta$ ainsi que les coordonnées du point $P^{*}$ obtenus par GRACE et par la méthode de surface de réponse proposée dans (Duprat et Sellier, 2006), notée SR, sont bien similaires, même si les différences ne sont pas négligeables. Elles peuvent être attribuées à une approximation imparfaite de la réponse réelle de la fonction de défaillance par la surface de réponse. La convergence obtenue par la surface de réponse est $a$ contrario meilleure, ce qui s'explique par le fait que l'algorithme de détermination de l'indice de fiabilité (ici HLRF) manipule un polynôme dont les dérivées partielles sont calculées analytiquement. Comme dans l'exemple précédent, le principal intérêt de GRACE est, pour une convergence satisfaisante, de l'obtenir à coût numérique 
réduit, par économie ici de 10 réévaluations des dérivées partielles de la fonction d'état limite (voir tableau 16).

\begin{tabular}{|c|c|c|c|c|c|}
\hline Technique & $\beta$ & $\mathrm{N}_{\mathrm{c}}$ & $\left|G\left(u^{*}\right) / G\left(u_{0}\right)\right|$ & $\left\|u^{*}-\alpha^{* T} u^{*} \alpha^{*}\right\|$ & $\max \left|u_{i}^{*}-u_{i\left(k^{*}-1\right)}\right|$ \\
\hline SR & 3,998 & 43 & $3,1710^{-4}$ & $8,70210^{-6}$ & $9,6210^{-3}$ \\
GRACE & 3,923 & 26 & $4,4810^{-4}$ & $1,31810^{-5}$ & $1,5410^{-2}$ \\
\hline
\end{tabular}

Tableau 14. Indice de fiabilité et convergence - Exemple 4

Pour cet exemple, la technique HLRF a été également appliquée sans procédure de prise en compte de l'imprécision numérique pour le calcul des dérivées partielles par différence finie : la perturbation appliquée pour déterminer l'incrément $\Delta x_{i}$ est une fraction de l'écart type $\sigma_{i}$, soit $\Delta x_{i}=\sigma_{i} / 1000$. On constate dans le tableau 17 que la convergence n'est pas atteinte pour le même nombre d'itérations (ni même au-delà). Cela s'explique par l'imprécision du calcul des gradients : la variation de la réponse sur la fonction d'état limite reste inférieure à la précision du résultat sur cette fonction, pour l'incrément considéré sur certains axes. Au-delà de la précision sur l'indice de fiabilité, de l'appartenance du point $P^{*}$ à la surface d'état limite, c'est la convergence même de l'algorithme qui est conditionnée par le contrôle d'erreur. Il convient également de souligner que seul l'aspect numérique est à l'origine de la non convergence de l'algorithme classique, et non la forme de la fonction d'état limite, suffisamment régulière et sans discontinuité au voisinage de $P^{*}$, conditions généralement nécessaires à la convergence des algorithmes basés sur le gradient projeté.

\begin{tabular}{|c|c|c|c|c|c|}
\hline Technique & $u^{*}{ }_{1}$ & $u^{*}{ }_{2}$ & $u^{*}{ }_{3}$ & $u^{*}{ }_{4}$ & $u^{*}{ }_{5}$ \\
\hline SR & 3,601 & 0,131 & $-0,0009$ & $-1,731$ & 0,016 \\
GRACE & 3,338 & $-0,078$ & $-0,025$ & $-2,026$ & 0,048 \\
\hline
\end{tabular}

Tableau 15. Coordonnées de $P^{*}$ - Exemple 4

\begin{tabular}{|c|c|c|c|c|c|c|c|}
\hline \multicolumn{2}{|c|}{ Itération } & 1 & 2 & 3 & 4 & 5 & 6 \\
\hline \multirow{4}{*}{ Variable } & $u_{1}$ & oui & oui & oui & oui & oui & oui \\
& $u_{2}$ & oui & oui & oui & oui & non & non \\
& $u_{3}$ & oui & oui & non & non & non & non \\
& $u_{4}$ & oui & oui & oui & oui & oui & oui \\
& $u_{5}$ & oui & oui & non & non & non & non \\
\hline
\end{tabular}

Tableau 16. Evaluation des dérivées partielles - Exemple 4 


\begin{tabular}{|c|c|c|c|c|c|c|c|}
\hline \multicolumn{2}{|c|}{ Itération } & 1 & 2 & 3 & 4 & 5 & 6 \\
\hline \multirow{2}{*}{ GRACE } & $\beta$ & 3,298 & 5,210 & 3,951 & 3,984 & 3,926 & 3,923 \\
& $\left|G\left(u^{*}\right) / G\left(u_{0}\right)\right|$ & 0,375 & 0,220 & 0,026 & 0,016 & 0,0006 & 0,0004 \\
\hline \multirow{2}{*}{ HLRF } & $\beta$ & 3,741 & 3,852 & 1,893 & 1,608 & 3,687 & 4,901 \\
& $\left|G\left(u^{*}\right) / G\left(u_{0}\right)\right|$ & 1,032 & 0,316 & 0,126 & 1,011 & 0,695 & 0,548 \\
\hline
\end{tabular}

Tableau 17. Comparaison de la convergence - Exemple 4

\section{Conclusion}

Dans cette étude, une méthode simple et efficace a été proposée pour permettre l'utilisation de l'algorithme de Rackwitz-Fiessler dans le cas où le modèle associé à la fonction de défaillance ne peut être défini qu'avec une certaine précision, ce qui est par exemple le cas pour les modèles aux éléments finis. Une procédure intégrée dans l'algorithme de Rackwitz-Fiessler, dénommée GRACE, tient compte de la précision numérique du modèle physique pour le choix de l'incrément de calcul nécessaire à l'estimation des dérivées partielles de la fonction d'état limite par différences finies. Elle permet d'une part, d'estimer correctement le vecteur gradient utilisé par l'algorithme de Rackwitz-Fiessler, et, d'autre part, d'économiser des calculs de la fonction d'état limite (en ne réévaluant pas systématiquement les dérivées partielles pour les variables aléatoires auxquelles le modèle est peu sensible). Les résultats obtenus sur quatre exemples issus de la littérature montrent bien l'intérêt de la méthode proposée. La convergence et la proximité du point final à la surface d'état limite restent satisfaisantes, même lorsque la fonction d'état limite présente un comportement fortement non linéaire et/ou un grand nombre de variables aléatoires. Le coût numérique de GRACE est, pour les exemples présentés, moindre que celui des autres techniques les plus usitées en fiabilité structurale. On peut également noter que tout algorithme dérivé de celui de Rackwitz-Fiessler, visant à en améliorer la précision et la convergence et s'appuyant sur la connaissance des gradients de la fonction d'état limite, comme ceux d'AbdoRackwitz et de Zhang-Der Kiureghian, pourrait tirer avantage de la procédure proposée.

\section{Remerciements}

Le CEA est remercié pour la mise à disposition du logiciel Cast3m dans sa version développement éducation et recherche. 


\section{Bibliographie}

Abdo T., Rackwitz R., "A new $\beta$-point algorithm for large time-invariant and time-variant reliability problems", Proc. of the $3^{\text {rd }} W G$ 7.5, Der Kiureghian A. and Thoft-Christensen P. eds., 1991.

Bonnans J.F., Gilbert J.-C., Lemarechal C., Sagastizabal C., Optimisation numérique : aspects théoriques et pratiques, Springer, collection Mathématiques et Applications, 1999.

Bucher C.G., Bourgund U., "A fast and efficient response surface approach for structural realiability problems", Structural Safety, vol. 7, 1990, p. 57-66.

Castem, http://www-cast3m.cea.fr, Version 2005.

Comrel, http://www.strurel.de, Version 8.00, 2008.

Duprat F., Sellier A., "Probabilistic approach to corrosion risk due to carbonation via an adaptive response surface method”, Probabilistic Engineering Mechanics, vol. 21, 2006, p. 207-216.

Hasofer A. M., Lind N. C., "An exact and invariant second moment code format", Journal of Engineering Mechanics, vol. 100, 1974, p. 111-121.

Haukaas T., Der Kiureghian A., "Strategies for finding the design point in non-linear finite element reliability analysis”, Probabilistic Engineering Mechanics, vol. 21, 2006, p. 133-147.

Liu P. L., Der Kiureghian A., Optimization algorithms for structural reliability analysis, Report UCB/SESM-86/09, University of California, Berkeley, 1986.

Liu P. L., Der Kiureghian A., "Optimization algorithms for structural reliability”, Structural Safety, vol. 9, 1991, p. 161-177.

Madsen H. O., Krenk S., Lind N.C., Methods of structural safety, Prentice-Hall, 1986.

Madsen H. O., “Omission sensitivity factors”, Structural Safety, vol. 5, 1988, p. 35-45.

Nataf A., «Détermination des distributions dont les marges sont données », Comptes-rendus de l'Académie des sciences, $\mathrm{n}^{\circ} 225,1962$, p. 42-43.

Nelder J.A., Mead R., “A simplex method for function minimization”, Comput. Journal, vol. 7, 1965, p. 308-313.

Nguyen X.S., Sellier A., Duprat F., Pons G., «Une méthode de surface de réponse adaptative en fiabilité des structures basée sur la régression pondérée », Revue européenne de mécanique numérique, vol. 16, 2007, p. 53-77.

OpenSees, http://opensees.berkeley.edu/index.php, Version 1.7.5, 2008.

Polak E., Optimization - Algorithms and consistent approximations, Applied Mathematical Sciences, Springer Verlag, vol. 124, 1997.

Rackwitz R., Fiessler B., "Structural reliability under combined random load sequences", Computers \& Structures, vol. 9, 1978, p. 489-494.

Zhang Y., Der Kiureghian A., "Two improved algorithms for reliability analysis", Proc. of the $6^{\text {th }}$ IFIP WG 7.5, Rackwitz R., Augusti A. and Borri A. eds., 1995.

Article reçu le 11 juin 2007 Accepté après révisions le 12 juillet 2008 